\title{
Closed Bipolar Electrochemistry for the Detection of Human Immunodeficiency Virus Short Oligonucleotide
}

\author{
Roghayeh Poorghasem, Reyhaneh Sadat Saberi, Mohsen Shayan, Masoud A. Mehrgardi*, \\ Abolfazl Kiani \\ Department of Chemistry, University of Isfahan, Isfahan, 81746-73441, Iran
}

\section{A R T I C L E I N F O}

\section{Article history:}

Received 30 August 2016

Received in revised form 18 November 2016

Accepted 22 November 2016

Available online 23 November 2016

\section{Keywords:}

Bipolar electrochemistry

Electrochemiluminescence

HIV1 virus

Electrochemical genosensor

\begin{abstract}
A B S T R A C T
Although a monumental progress has been made in case of Human Immunodeficiency Virus researches, there are still more than 34 million people living with this viral disease. Therefore, the development of a fast and cost-effective protocol that can sensitively and selectively detect HIV gene sequence is essential. In the present study, a protocol for the molecular detection of HIV genome taking advantage of closed bipolar electrochemistry integrated with electrogenerated chemiluminescence detection is described. In this case, a closed split bipolar electrochemical cell is applied to detect the short specific oligonucleotide target of HIV1. Anthraquinone disulfonic acid, as a negative electroactive reporter, intercalates with hybridized double-strand DNA on the sensing site. By reduction of redox reporter (AQDS) on the cathodic pole, luminol is oxidized on the anodic pole and subsequent oxidized diazo product generates light that is followed using a photomultiplier tube. The experimental condition is optimized to obtain the highest sensitivity. The results demonstrate the high sensitivity and selectivity of this fast and economical method toward Human Immunodeficiency Virus genome detection in the range of 0.1 to $300 \mathrm{nM}$ with the detection limit of $30 \mathrm{pM}(\mathrm{S} / \mathrm{N}=3)$.
\end{abstract}

(c) 2016 Elsevier Ltd. All rights reserved.

\section{Introduction}

Human Immunodeficiency Virus (HIV) is the virus responsible for Acquired Immunodeficiency Syndrome (AIDS). AIDS is spreading in different countries worldwide and unfortunately statistics show that there are more than 34 million people infected by this fatal virus [1]. Therefore, there is a high demand for a sensitive and selective method that can simply and easily detect HIV genome. To date, HIV is divided into HIV-1 and HIV-2 strains [2], whereas HIV-2 represents a significant minority of all HIV infections [3]. Traditional methods for DNA detection like gel electrophoresis and currently available diagnostic technologies like PCR-based methods are slow and time consuming. DNA hybridization biosensors provide valuable sequence-specific information with a faster, simpler and economical approach in comparison to the traditional protocols. There are plenty of available diagnosis technologies for HIV genome based on Fluorescence [4] electrochemical methods [5,6], electrochemiluminescence [7] and etc.

\footnotetext{
* Corresponding author. Tel.: +98 3137934940; fax: +98 3136689732.

E-mail addresses: m.mehrgardi@chem.ui.ac.ir, m.mehrgardi@gmail.com (M.A. Mehrgardi).
}

Bipolar electrochemistry (BE) consists of a driving potential applied to a pair of driving electrodes through an electrolyte solution containing conducting object (bipolar electrode; BPE) $[8,9]$. The driving potential drops in the solution and therefore induces a potential differences along the BPE. In a sufficient potential difference, the faradic reactions simultaneously occur at the both ends of BPE. Detailed of bipolar electrochemistry and applications are recently reviewed [10-12]. In the last decade, bipolar electrochemistry is revived for analytical and bioanalytical purposes and surface modification in nano or micro scales $[9,13-$ 18].

Electrogenerated chemiluminescence (ECL) is a mean that emits measurable luminescent signals by converting electrochemical energy into radiative energy via an electrochemical reaction [19]. ECL is known as a fast, domestic, sensitive analytical method which can be integrated with various techniques. Since there is no need for a light source, the most significant feature of ECL is low background, high sensitivity and very low detection limit (around $10^{-11}$ Molecule/L) [20,21]. Moreover, ECL has the features of time and spatial resolution in which time and position of the emitted light could be under control [22]. More details about this technique and all the recent advances are reviewed by [23]. Lately, ECL is very appealing in biosensor application [24,25]. 
Integration of $\mathrm{BE}$ and $\mathrm{ECL}$ detection has attracted a lot of attention [26,27]. This technique is used for the first time in 2001 by Manz's group [28] by taking the advantages of ECL as a detector for electrokinetic chromatographic separation. Our research group, recently developed a visualized protocol for the detection of single nucleotide polymorphisms based on wireless ECL bipolar system [29]. Coupling BE and ECL detection method leads to a sensitive, selective, low-cost biosensor for high throughput applications and simple and rapid diagnostics [27,30]. The wireless nature of BPE and no need for a light source in ECL simplifies the analytical set up to a great extent.

A popular system for ECL at positive potentials is luminol and its co-reactant, hydrogen peroxide, under variety of experimental conditions [31,32]. This ECL system has been applied to many biosensor applications for the detection of cancer biomarkers [33], thrombin [34], immunoglobulin G [35] and etc.

In this study, a genosensor has been designed and fabricated based on integrated closed split BE-ECL system for the detection of HIV1 short oligonucleotide sequence. AQDS as a negative electroactive redox intercalator was applied on the sensing site. While AQDS reduces on the cathodic pole, luminol was oxidized on the anodic pole and ECL signal was monitored by a photomultiplier tube (PMT). The present genosensor shows good analytical performances with a dynamic range of 0.1 to $300 \mathrm{nM}$ with a great detection limit of 30 pM toward HIV1 detection.

\section{Experimental}

\subsection{Materials}

Nitric acid, sodium dihydrogen phosphate, disodium hydrogen phosphate, sodium chloride, magnesium chloride, sodium hydroxide, potassium chloride, Potassium ferrocyanide, Potassium ferricyanide, 6-mercapto 1-hexanol $(\mathrm{MCH})$, sodium carbonate, Tris-(2-carboxyethyl) phosphine (TCEP), 9,10-Anthraquinone-2,6disulfonic acid disodium (AQDS), 5-amino-2-3-dihydro-1-4phthalazindione (luminol) and hydrogen peroxide were purchased from commercial sources (Merck or Sigma) and selected in analytical grade and used as received, without further purification. The distilled-deionized water was used in all solution preparation.

The stock solutions of the oligonucleotides were prepared using PBS buffer and kept frozen at $-20^{\circ} \mathrm{C}$. In this study, DNA oligonucleotides were obtained from Eurofins MWG Operon Co. (Germany) and had the following sequence. The hairpin sequence as the probe and its complementary target were adopted from [36] (From $5^{\prime}$ to $3^{\prime}$ ):

Probe: GCGAGCCTGGGATTAAATAAAATAGTAAGAATGTATAGCGCTCGC- $\left(\mathrm{CH}_{2}\right)_{6}-\mathrm{SH}$

Complementary: GCTATACATTCTTACTATTTTATTTAATCCCAG

Basically, the probe sequences are selected to contain a part of the target genome which is not similar to any of the human genomes in the healthy cells.

\subsection{Instrumentation:}

The potential on the driving electrodes of $\mathrm{BE}$ system was applied using a Mastech DC power supply (HY3005F-3). A homemade chemiluminescence detector, consisted of a photomultiplier tube (PMT) R928 model from Hamamatsu (Japan), a high-voltage DC power supply, a current to voltage follower, an amplifier, and analog to digital converter (Arman Optimized Systems, Iran).

Electrochemical impedance spectroscopy (EIS) and cyclic voltammetry (CV) experiments were performed with an Autolab PGSTAT30 (ECO Chemie, Netherlands) and $\mu$ Autolab PGSTAT101 (ECO Chemie, Netherlands) potentiostat/galvanostat using NOVA, PGES 4.9 and FRA 4.9 software in a three-electrode system containing a small piece of an archival gold compact discrecordable (Pro Gold Archival CD-R, Memorex, USA) as a working electrode, an $\mathrm{Ag} / \mathrm{AgCl} / 3.0 \mathrm{M} \mathrm{KCl}$ as a reference electrode and a platinum wire as an auxiliary electrode. EIS experiments were carried out at open circuit potential (OCP) by applying an AC potential with a signal amplitude of $5 \mathrm{mV}$ and a frequency in the range of $10 \mathrm{kHz}$ to $0.1 \mathrm{~Hz}$ and in the presence of $0.5 \mathrm{mM}$ $\mathrm{Fe}(\mathrm{CN})_{6}{ }^{3-/ 4-}$ redox probe (in $0.1 \mathrm{M} \mathrm{KCl}$ ) in OCP mode.

The BE-ECL experiment was performed in a rectangular cell made of Teflon. The cell was divided into two chambers (Fig. 1a and b) with dimensions of $60 \times 7 \mathrm{~mm}$ and depth of $4 \mathrm{~mm}$. There are four holes for driving electrode placement with dimensions of $15 \times 13 \mathrm{~mm}$ and depth of $12 \mathrm{~mm}$. the cell volume is $10 \mathrm{~mL}$. During the BE-ECL measurement the cell was covered with a lid and an optical fiber with $1.2 \mathrm{~mm}$ diameter was inserted in a hole on the top of the lid in anodic section to transfer the ECL signal to the detector.

\subsection{Closed Split BE-ECL Cell}

The ECL reaction on the anodic pole requires alkaline medium which is completely different with the optimized conditions for

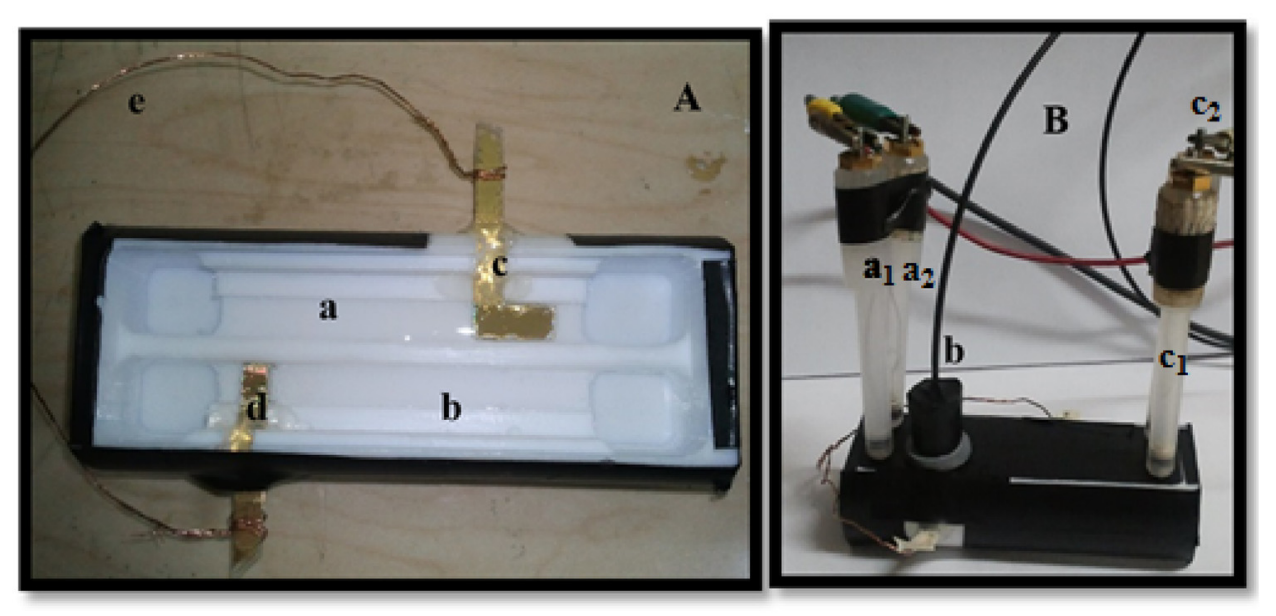

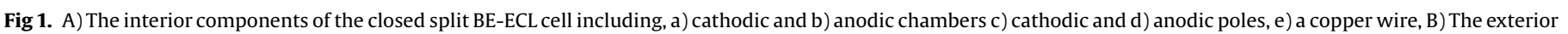
components of the same cell after closing the lid including, $a_{1}, a_{2}, c_{1}$ and $c_{2}$ ) four driving electrodes and b) a fiber optic. 
the stability of DNA in the duplex form. Therefore, a closed split bipolar cell has been designed as shown in Fig. 1. The bipolar electrode was divided in two separate poles, located at cathodic (Fig. 1A, a) and anodic (Fig. 1A, b) chambers. The chambers containing cathode and anode poles (Fig. 1A, c and d) were separated by a wall and two electrodes were connected using a conducting wire (Fig. 1A, e) to keep electrical connection and the bipolar performance. According to Zhan et al. research on the surface ratio effect of two split poles on the ECL efficiency, the L-shaped cathodic pole was designed which provides a high surface ratio of two divided poles and promotes the electron transfer yield from anodic to cathodic pole [37]. The two-driving electrode set up in bipolar electrochemistry cannot be applied to this closed split bipolar cell due to the mixing of alkaline and buffer solution. Therefore, each driving electrode in each side, split in two (Fig. 1B, a1, a2 and $\mathrm{c} 1, \mathrm{c} 2$ ) and each piece was placed in an individual chamber. By the aim of this set up, each piece of driving electrode worked as a separate driving electrode. The same voltage to the split pieces at one side was applied, the overall performance of the split driving electrodes is the same as the non-split one.

\subsection{Modification of cathodic pole using a short sequence of HIV1 virus genome}

As mentioned above, the bipolar electrodes were prepared using small pieces of archival compact disk (CD) with gold reflective layer as previously reported in the literature [38]. Briefly, a piece of $C D$ was cut and the protective layer was removed by putting it in the concentrated nitric acid solution. Then it was washed with water thoroughly. Now the gold surface was exposed and it could be used as anodic and cathodic poles. Usually, the chemical attack to the protective films required just two minutes and after that, the remaining material can be easily removed by water. The surface of the gold electrode was electrochemically cleaned in $0.5 \mathrm{M}$ of sulfuric acid by potential cycling over 0 to $1.5 \mathrm{~V}$. TCEP was used to deprotect the disulfide end of probe DNA to thiol form. Therefore, $20 \mu \mathrm{L}$ probe DNA $(5 \mu \mathrm{M})$ was treated by $6.0 \mu \mathrm{L}$ of $0.5 \mathrm{mM}$ TCEP in PBS (pH 7.4). The above solution was kept in $4^{\circ} \mathrm{C}$ for 1 hour. Then, $12 \mu \mathrm{L} \mathrm{MgCl}_{2}$ solution $(3 \mathrm{mM}$ ) was added to this solution to minimize the repulsion between the strands on the surface. Subsequently, the prepared solution was casted on the cathodic pole surface overnight. To remove non-specific adsorbed thiolated probes to the surface, the electrode was then rinsed by PBS buffer and treated with $6.0 \mu \mathrm{L} \mathrm{MCH}(100 \mathrm{nM})$ for 10 minutes. For hybridization step, the modified cathodic pole surface was exposed to $20 \mu \mathrm{L}$ of the complementary target containing magnesium chloride, again to minimize the repulsion and improve hybridization efficiency, for three hours. After rinsing the surface by buffer, it was dipped in $1 \mathrm{mM}$ AQDS as an electroactive reporter and mounted on the cell.

After mounting the modified cathodic pole, the anodic pole was then fixed in the other chamber of the cell. The cathodic chamber was filled with $10 \mathrm{mM}$ PBS solution. The anodic chamber was filled with $1.0 \mathrm{mM}$ luminol solution containing hydrogen peroxide $0.1 \mathrm{M}$ and sodium carbonate $0.25 \mathrm{M}, \mathrm{pH} 12.5$. In the last step, the cell was covered by a lid. An optical fiber with $1.2 \mathrm{~mm}$ diameter was inserted in a hole on the top of the lid in anodic section to transfer the ECL signal to the detector. The ECL signal has been followed by averaging the signals over $0.1-0.15 \mathrm{~s}$.

\section{Results and Discussion}

In this research, a short sequence of HIV1 genome, adapted from [36], was detected by a sensitive, simple and selective biosensor based on a closed split BE system to separate the cathodic and anodic chambers of the BE cell and avoid the mixing of different electrolyte. The BE-ECL measurement has been performed. Different steps of cathodic pole modification and ECL measurement has been demonstrated in Scheme 1. In a closed BE system, the solutions contacting the BPE anode and cathode are physically separated from each other and the current passes between the two half cells through the electrodes. Although most of the BPEs are continuous, it is also possible to connect two or more electrodes to make a split one. The advantage of split bipolar electrodes over continuous design is the easier voltage measurement between the anodic and cathodic poles, just by connecting a voltmeter to the external circuit. Furthermore, the cathodic pole had to be pretreated before modification using cyclic voltammetry in sulfuric acid solution which required a connection to the electroanalyzer instrument.

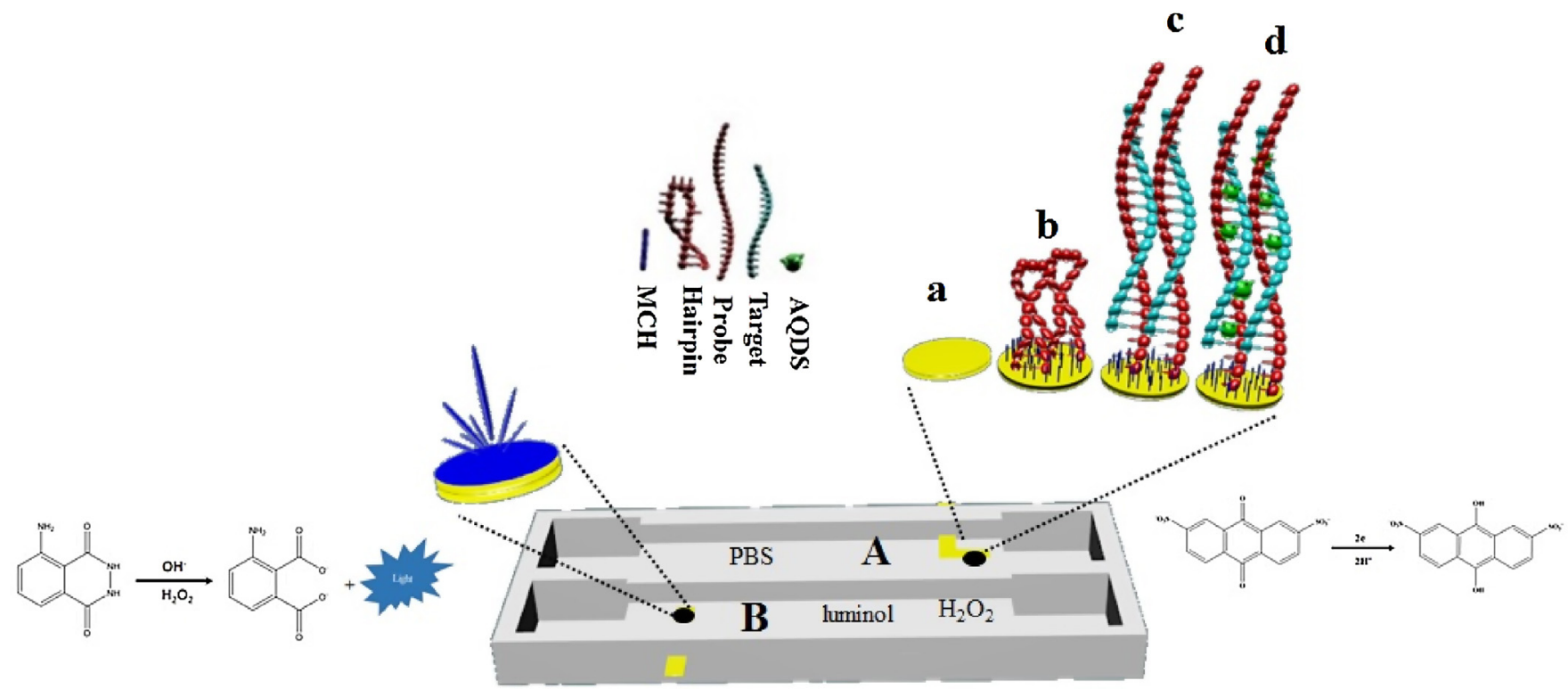

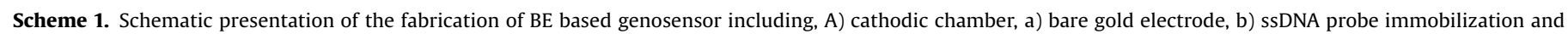
blocking with $\mathrm{MCH}, \mathrm{c}$ ) Hybridization with the target and e) AQDS intercalation. B) Anodic Chamber and the final ECL response of luminol. 
A short sequence of HIV genome was immobilized on the cathodic pole. Since some nucleotides at the probe are complementary to some others in the same oligonucleotide, the probe DNA can form a hairpin (Scheme 1A, b). Subsequently, it was hybridized with the complementary DNA target. The formed DNA duplex is a trap for the intercalator molecule, Anthraquinone disulfonic acid (AQDS) (Scheme 1A, c and d). AQDS is a negative redox probe which reduces at the surface as following:

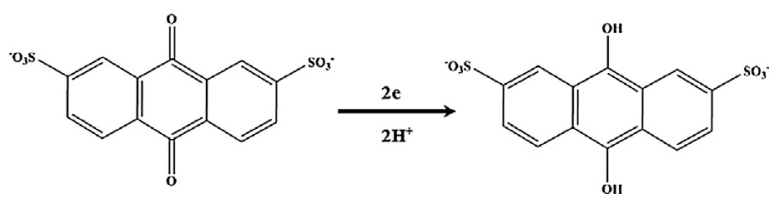

On the other side, the anodic chamber was filled with luminol and hydrogen peroxide solution in an alkaline $\mathrm{pH}$. The reduction of the redox probe intercalated in hybridized ds-DNA, induces the luminol oxidation on anodic pole at the other chamber of the cell (Scheme 1B). The ECL signal of luminol depends on the concentrations of the target.

\subsection{BE-ECL Optimized Design}

As mentioned in the experimental section, a BE-ECL was designed to fulfill the experimental requirements (Fig. 1). The anodic and cathodic poles were made of a treated recordable compact disk (CD) [38]. The gold layer of CD on the cathodic pole was modified with a short sequence of HIV1 virus genome. Two separated chambers for mounting the cathodic and anodic electrodes has been designed in the split closed cell to not disturb the stability of duplex form of the immobilized DNA due to alkaline solution required for ECL reaction on the other pole. The cell also consisted of anodic and cathodic poles of the bipolar system, four driving platinum electrodes (two electrodes for each chamber) and a power supply. The two-driving electrode set up in bipolar electrochemistry cannot be applied to this closed split bipolar cell due to the mixing of alkaline and buffer solution. Therefore, each driving electrode in each side, split in two (Fig. 1B, a1, a2 and c1, c2) and each piece was placed in an individual chamber. By the aim of this set up, each piece of driving electrode worked as a separate driving electrode. The same voltage to the split pieces at one side was applied. The overall performance of the split driving electrodes is the same as the non-split one. One of the main challenges in BE systems is the different optimized conditions for the anodic and cathodic reactions. This design allowed us to overcome this issue and to use two different electrolytes in the anodic and the cathodic chamber even with the different pHs. On the other side, in this design, the same potential gradients were forced in each chamber between the driving electrodes, responsible for the performing of bipolar reactions. Moreover, by considering the same distance between the driving electrodes, both chambers felt the same electric field. Based on the previous investigations on the effect of the shape and surface area of the cathodic and anodic poles on the signal intensity [37], the L-shaped cathodic pole has been used in this research and caused to prompt the electron transfer yield from anodic to cathodic pole.

In the most of BE-ECL systems, $\mathrm{Ru}(\mathrm{bpy})_{3}{ }^{2+}$ has been applied as the luminophore [10] but in the present manuscript, luminol was chosen. $\mathrm{Ru}(\mathrm{bpy})_{3}{ }^{2+}$ and luminol as two common luminophores have some advantages and disadvantages. Although, luminol is consumed and $\left[\mathrm{Ru}(\mathrm{bpy})_{3}\right]^{2+}$ can be regenerated at the anodic pole, ECL of luminol requires lower potentials [39] and is faster and cheaper than $\mathrm{Ru}(\mathrm{bpy})_{3}{ }^{2+}[40]$. After mounting the electrodes in the cell, the cathodic and anodic chambers were filled with $10 \mathrm{mM}$ PBS solution ( $\mathrm{pH} 7.0$ ) and a solution of luminol and hydrogen peroxide

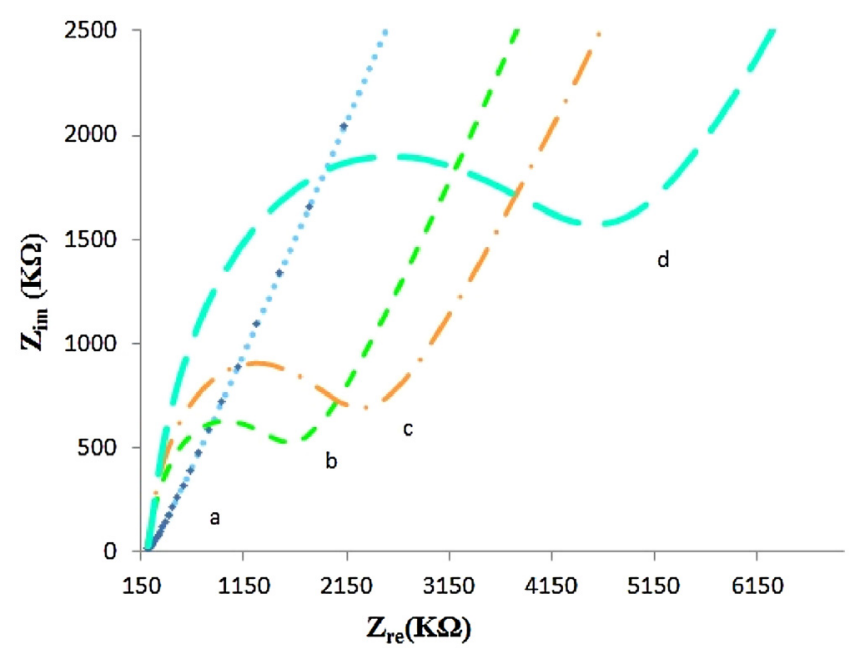

Fig. 2. The Nyquist plots obtained in $0.5 \mathrm{mM} \mathrm{Fe}(\mathrm{CN})_{6}{ }^{3-/ 4-}$ containing $0.1 \mathrm{M} \mathrm{KCl}$ at OCP for a) the bare cathodic pole, and each step of modification with b) ssDNA probe, c) blocking with the $\mathrm{MCH}, \mathrm{d}$ ) hybridization with the complementary target.

( $\mathrm{pH}$ 12.5), respectively. Then two platinum electrodes, located on one side of each chamber, were connected to the positive pole of the power supply and other two platinum electrodes on the other side were connected to the negative pole. Finally, the fiber optic was fixed on the cell cover to transfer ECL emission to the detector.

\subsection{Characterization of the modified layers on cathodic pole by Electrochemical Impedance spectroscopy}

Electrochemical Impedance spectroscopy (EIS) was used as a powerful electrochemical method to investigate the surface of gold cathodic pole after each step of modification. Fig. 2 illustrates the Nyquist plots of bare, thiolated single stranded HIV1 probe modified gold electrode, and after the hybridization of the immobilized probe on the surface with the target in equimolar potassium ferri/ferrocyanide solution $(0.5 \mathrm{mM})$. As it is shown in

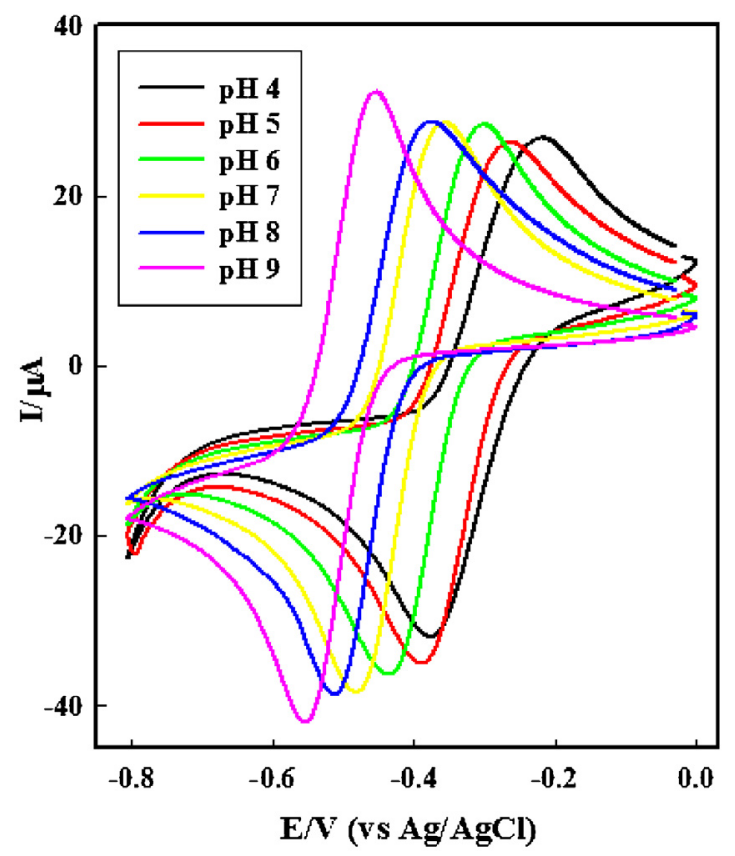

Fig. 3. Cyclic voltammograms of AQDS $1.0 \mathrm{mM}$ in different pHs over 4.0 to 9.0 on a bare gold electrode with scan rate $100 \mathrm{mV} \mathrm{s}^{-1}$. 
Fig. 2, the charge transfer resistance $\left(R_{c t}\right)$ for the bare gold electrode is $\sim 250 \mathrm{ohm}$ indicating the fast charge transfer at the bare electrode (Fig. 2a). After immobilization of the probe on the surface of the gold electrode, $\mathrm{R}_{\mathrm{ct}}$ is increased and charge transfer occurs slower to the $\mathrm{Fe}(\mathrm{CN})_{6}{ }^{3-} / \mathrm{Fe}(\mathrm{CN})_{6}{ }^{4-}$ (Fig. 2b). The decrease in the charge transfer kinetic may be attributed to the repulsion between the negatively charged iron probe and negatively charged phosphate backbone of the probe on the surface. Hybridization of the probe with complementary target develops the negative charges on the surface and causes more increase in the $\mathrm{R}_{\mathrm{ct}}$ (Fig. 2d)

\subsection{Investigation of experimental parameters on the ECL signal}

The effect of several experimental parameters on the intensity of the ECL signal were studied including: $\mathrm{pH}$ and applied voltage. The advantages of using negatively charged redox probes for DNA biosensors have been described in details in previously published manuscripts [41,42]. Application of the negative probes overcomes two main challenges in DNA biosensors, first and the foremost, the false positive signal for the target with a few mismatches due to the intercalation of the probe close to the electrode surface and
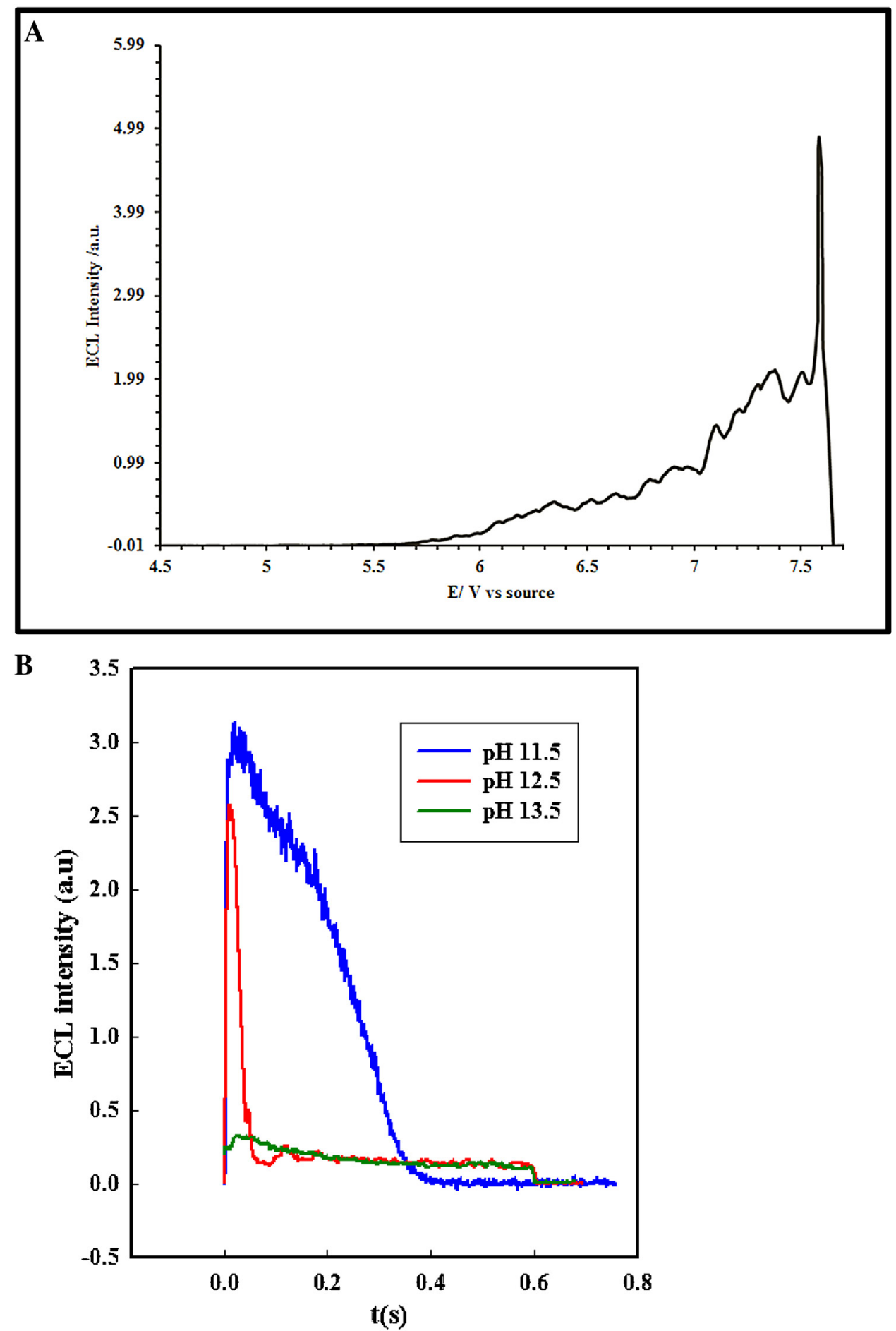

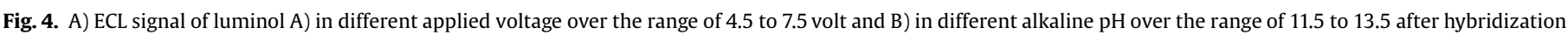
with complementary target. 
moreover, the false positive signal of penetration of the probe through SAM pinholes. Therefore, in this research, AQDS was chosen as a negative redox reporter which is oxidized and reduced at in $-0.35 \mathrm{~V}$ and $-0.45 \mathrm{~V}$ at $\mathrm{pH} 7.0$, respectively.

One of the most important parameters on signal intensity of the bipolar electroluminescence is the AQDS reduction on cathodic pole. To investigate the $\mathrm{pH}$ effect on electrochemical behavior of AQDS, the cyclic voltammograms of $1.0 \mathrm{mM}$ AQDS was taken in various pHs over range of 4 to 9 (Fig. 3). As the figure shows, by increasing the $\mathrm{pH}$, the oxidation and reduction potentials of AQDS are shifted toward more negative voltages, but the intensity of the anodic and cathodic currents do not change significantly. There are two main challenges for applying high negative potentials to the surface of BPEs. First and the foremost, disassembly of thiolated monolayer from the gold surface and moreover, oxygen reduction. To avoid these issues and also for the stability of the dsDNA, biological $\mathrm{pH}$ has been selected as the optimized $\mathrm{pH}$.

To determine the least required voltage to trigger ECL reaction of luminol on anodic pole and AQDS reduction on probe modified cathodic pole, the various potentials over range of 4.5 to $7.5 \mathrm{~V}$ have been applied to the driving electrodes (Fig. 4A). The results demonstrate the emission of ECL starts $\sim 5.5 \mathrm{~V}$ and higher voltages enhance the ECL intensities. The applied potential to the driving electrodes by power supply is not as same as the sensed potential by the bipolar electrodes. The potential drops through the electrolyte solution and the higher applied potentials on the driving electrodes cause more positive potentials on the anodic pole and more negative potentials on the cathodic pole. Regarding the above fact, the potential between cathodic and anodic poles of bipolar system was checked each time by a voltmeter after changing the applied potential on the driving electrodes. As it was mentioned above, more negative potentials can disassemble the probe monolayer on the surface. There is a linear relation between the applied potential to the driving electrodes and the potential that is sensed at the poles. When the potential of $6.0 \mathrm{~V}$ is applied to the system, the poles sense the potential around $1.0 \mathrm{~V}$ to $1.2 \mathrm{~V}$. This potential is enough for electrochemical reduction of AQDS and oxidation of luminol. While by increasing the voltage, the ECL intensity has been increased but at higher potentials, the microbubbles around the cathodic pole were observed that obviously demonstrates the reduction of water on the surface. Therefore, the potential $6.0 \mathrm{~V}$ was selected as the optimized potential for the ECL
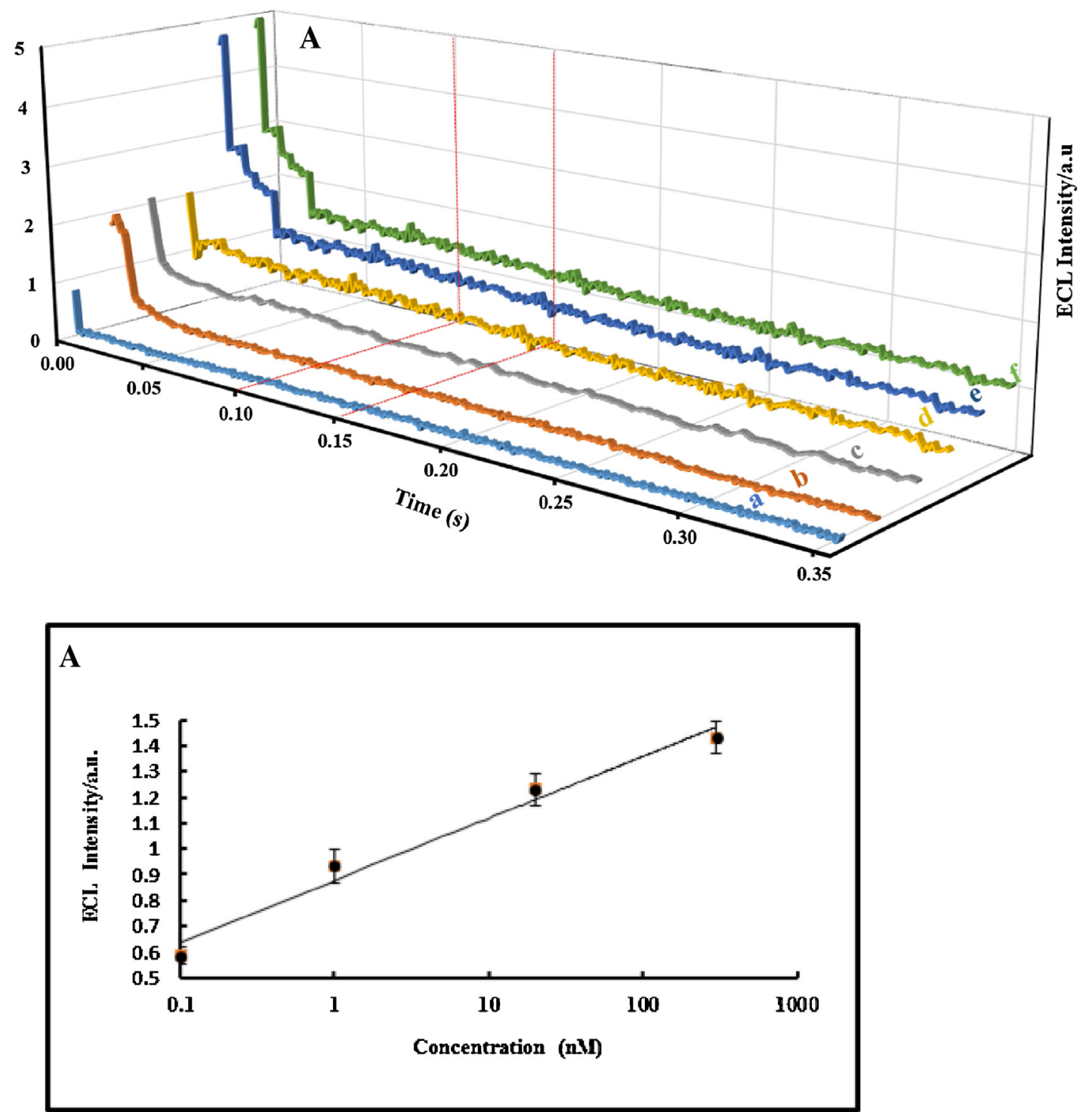

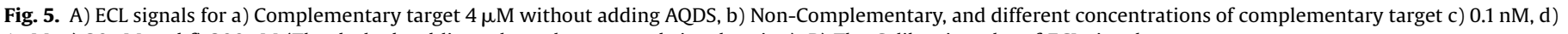
$1 \mathrm{nM}$, e) $20 \mathrm{nM}$ and f) $300 \mathrm{nM}$ (The dashed red lines show the averaged signal region). B) The Calibration plot of ECL signal vs target amount. 
reaction. Luminol chemiluminescence has the highest yield in $\mathrm{pH}$ 12.5 (Fig. 4B) [43]. Therefore, the anodic chamber was filled with optimized alkaline $\mathrm{pH} 12.5$ for all the ECL experiments.

\subsection{The Analytical Performances of the biosensor for HIV1 detection}

One of the key challenges in genosensors is the unreliable signal due to the single nucleotide mismatched targets. By engineering an appropriate probe structure, the ability of the genosensor toward discrimination between complementary and non-complementary targets can be increased to a great extent. Hairpin probe is an oligonucleotide with a structure made of a stem and a loop. This probe is very selective to complementary sequence, especially when in targets with one mismatched, the mutation is positioned within the loop sequence $[44,45]$. Therefore, the hairpin probe structure is more reliable for genetic analysis.

The probe modified cathodic pole was hybridized with different concentrations of complementary (Fig. 5A, c-f) and non-complementary targets. The control experiment for non-complementary target did not show any significant ECL signal expressing the selectivity of the signal (Fig. 5A, b). The complementary target without adding any redox probe as another control experiment revealed the accuracy of the ECL signal as well (Fig. 5A, a). After the formation of DNA in duplex form, it was treated with AQDS solution and then, the cathodic pole was rinsed carefully with phosphate buffer solution to remove the non-specific adsorbed redox probe molecules on the surface. By exposing the cathode surface to the target, the density of DNA duplex was enhanced at the surface of the cathode. DNA duplex is a trap for AQDS as an intercalator. The reduction of AQDS on the surface of the cathode triggers the oxidation of luminol and generates luminol ECL signal. ECL signal has been followed by averaging the signal over $0.1-0.15 \mathrm{~s}$. While the maximum signals show no significant differences, the differences between the signals in this region are obvious. There is a linear relation for ECL intensities versus the target amount (Fig. 5B).

The results show not only the high ability of the biosensor for sensitive detection of the target ssDNA, but also the high selectivity of the biosensor for discrimination between complementary and non-complementary sequences of target molecule over the range of 0.1 to $300 \mathrm{nM}$ with a detection limit of $30 \mathrm{pM}$ (based on $\mathrm{S} / \mathrm{N}=3$ ). For calculation of RSD, a solution of $4 \mu \mathrm{M}$ of the target was analyzed three times. RSD of $2.36 \%$ reveals the high precision of the measurement by this biosensor. According to the literature, most of the HIV biosensors are based on p24 antigen immunoassay [46,47] or HIV protease [48] measurement or enzyme and protein biosensor modifications which require special care and conditions [49]. A recent research has been made on HIV RNA target measurement which also suffer from RNA decomposition [50]. Obviously, this biosensor can easily beat the aforementioned devices in terms of simplicity and cheapness. Moreover, it has a privilege of great detection limit $(30 \mathrm{pM})$ for HIV genome measurement in comparison to the rivals [51,52]. In addition, the bipolar electrochemistry as a capable tool for detection of DNA hybridization in different concentrations of target, has the potential of measurement in high throughput sampling with wireless microarray electrodes.

\section{Conclusions}

A method for DNA hybridization detection of HIV1 short sequence is introduced by coupling BP and ECL and taking advantage of more reliable signal of negative AQDS probe and costeffective luminophore of luminol on a cheap, available recordable compact disk. The results demonstrate an economical and simple set up with the significant performance as a biosensor for sensitive and selective detection of the complementary target molecule.
Since a bipolar system integrated to the ECL system detection does not require any light source, it is capable of intensification in the signal to the noise ratio and the higher sensitivity toward the target by removing the common background emission or noises in chemiluminescence system.

\section{Acknowledgment}

We gratefully acknowledge the support of this work by the Research Council of University of Isfahan and Iran National science foundation (Grant No. 93031920).

\section{References}

[1] F. Barre-Sinoussi, A.L. Ross, J.-F. Delfraissy, Nat. Rev. Micro. 11 (2013) 877-883. [2] J.D. Reeves, R.W. Doms, Journal of General Virology 83 (2002) 1253-1265.

[3] T.I. de Silva, M. Cotten, S.L. Rowland-Jones, Trends in Microbiology 16 (2008) 588-595.

[4] Q. Cao, Y. Teng, X. Yang, J. Wang, E. Wang, Biosensors and Bioelectronics 74 (2015) 318-321.

[5] A. Rahim Ruslinda, K. Tanabe, S. Ibori, X. Wang, H. Kawarada, Biosensors and Bioelectronics 40 (2013) 277-282.

[6] M.A.M. Rodrigo, Z. Heger, N. Cernei, A.M.J. Jinemez, O. Zitka, V. Adam, R. Kizek, Int. J. Electrochem. Sci. 9 (2014) 3449-3457.

[7] S. Ruan, Z. Li, H. Qi, Q. Gao, C. Zhang, Mikrochim Acta 181 (2014) 1293-1300.

[8] N. Dorri, P. Shahbazi, A. Kiani, Langmuir 30 (2014) 1376-1382.

[9] F. Mavré, R.K. Anand, D.R. Laws, K.-F. Chow, B.-Y. Chang, J.A. Crooks, R.M. Crooks, Analytical Chemistry 82 (2010) 8766-8774.

[10] S.E. Fosdick, K.N. Knust, K. Scida, R.M. Crooks, Angewandte Chemie International Edition 52 (2013) 10438-10456.

[11] C.A.C. Sequeira, D.S.P. Cardoso, M.L.F. Gameiro, Chemical Engineering Communications 203 (2016) 1001-1008.

[12] L. Bouffier, S. Arbault, A. Kuhn, N. Sojic, Analytical and Bioanalytical Chemistry 408 (2016) 7003-7011.

[13] X. Zhang, C. Chen, J. Li, L. Zhang, E. Wang, Analytical Chemistry 85 (2013) 5335-5339.

[14] X. Zhang, J. Li, X. Jia, D. Li, E. Wang, Analytical Chemistry 86 (2014) 5595-5599.

[15] H.-W. Shi, W. Zhao, Z. Liu, X.-Ch. Liu, J.-J. Xu, H.-Y. Chen, Analytical Chemistry 88 (2016) 8795-8801.

[16] Y.-Z. Wang, W. Zhao, P.-P. Dai, H.-J. Lu, J.-J. Xu, J. Pan, H.-Y. Chen, Biosensors and Bioelectronics 86 (2016) 683-689.

[17] M.-S. Wu, Z. Liu, H.-W. Shi, H.-Y. Chen, J.-J. Xu, Analytical Chemistry 87 (2015) $530-537$.

[18] Huai-Rong Zhang, Yinzhu Wang, Wei Zhao, Jing-Juan Xu, Hong-Yuan Chen, Analytical Chemistry 88 (2016) 2884-2890.

[19] A.J. Bard, Electrogenerated Chemiluminescence, Marcel Dekker, New York, 2004.

[20] C. Bertolino, M. MacSweeney, J. Tobin, B. O’Neill, M.M. Sheehan, S. Coluccia, H. Berney, Biosensors and Bioelectronics 21 (2005) 565-573.

[21] A.-M. Spehar, S. Koster, S. Kulmala, E. Verpoorte, N. de Rooij, M. Koudelka-Hep, Luminescence 19 (2004) 287-295.

[22] L.S. Dolci, S. Zanarini, L.D. Ciana, F. Paolucci, A. Roda, Analytical Chemistry 81 (2009) 6234-6241.

[23] Z. Liu, W. Qi, G. Xu, Chemical Society Reviews 44 (2015) 3117-3142.

[24] Q. Sun, X. Zhang, J Solid State Electrochem 16 (2012) 247-252.

[25] H. Wei, E. Wang, Luminescence 26 (2011) 77-85.

[26] S.E. Fosdick, J.A. Crooks, B.-Y. Chang, R.M. Crooks, Journal of the American Chemical Society 132 (2010) 9226-9227.

[27] H.-W. Shi, M.-S. Wu, Y. Du, J.-J. Xu, H.-Y. Chen, Biosensors and Bioelectronics 55 (2014) 459-463.

[28] A. Arora, J.C.T. Eijkel, W.E. Morf, A. Manz, Analytical Chemistry 73 (2001) 32823288.

[29] S.M. Khoshfetrat, M. Ranjbari, M. Shayan, M.A. Mehrgardi, A. Kiani, Analytical Chemistry 87 (2015) 8123-8131.

[30] T. Wang, S. Fan, R. Erdmann, C. Shannon, Langmuir 29 (2013) 16040-16044.

[31] H. Cui, W. Wang, C.-F. Duan, Y.-P. Dong, J.-Z. Guo, Chemistry-A European Journal 13 (2007) 6975-6984.

[32] X. Liu, W. Niu, H. Li, S. Han, L. Hu, G. Xu, Electrochemistry Communications 10 (2008) 1250-1253.

[33] X. Jiang, H. Wang, R. Yuan, Y. Chai, Biosensors and Bioelectronics 63 (2015) 33-38.

[34] Y.-P. Dong, T.-T. Gao, Y. Zhou, J.-J. Zhu, Analytical Chemistry 86 (2014) 1137311379.

[35] L. Xiao, Y. Chai, R. Yuan, Y. Cao, H. Wang, L. Bai, Talanta 115 (2013) 577-582.

[36] J.A.M. Vet, A.R. Majithia, S.A.E. Marras, S. Tyagi, S. Dube, B.J. Poiesz, F.R. Kramer, Proceedings of the National Academy of Sciences 96 (1999) 6394-6399.

[37] W. Zhan, J. Alvarez, R.M. Crooks, Journal of the American Chemical Society 124 (2002) 13265-13270.

[38] L. Angnes, E.M. Richter, M.A. Augelli, G.H. Kume, Analytical Chemistry 72 (2000) 5503-5506.

[39] V. Eßmann, D. Jambrec, A. Kuhn, W. Schuhmann, Electrochemistry Communications 50 (2015) 77-80. 
[40] G. Jirka, T. Nieman, Mikrochim Acta 113 (1994) 339-347.

[41] M.A. Mehrgardi, R. Daneshtalab, Journal of Electroanalytical Chemistry 650 (2011) 214-218.

[42] E.L.S. Wong, J.J. Gooding, Analytical Chemistry 75 (2003) 3845-3852.

[43] A.L.S. Chandel, S.A. Khan, R.S. Kher, A. Tiwari, Luminescence 27 (2012) $455-458$.

[44] E. Farjami, L. Clima, K. Gothelf, E.E. Ferapontova, Analytical Chemistry 83 (2011) 1594-1602.

[45] S. Tyagi, F.R. Kramer, Nat. Biotech. 14 (1996) 303-308.

[46] J. Liu, B. Du, P. Zhang, M. Haleyurgirisetty, J. Zhao, V. Ragupathy, S. Lee, D.L.D. Voe, I.K. Hewlett, Biosensors and Bioelectronics 61 (2014) 177-183.
[47] P. Fan, X. Li, W. Su, W. Kong, X. Kong, Z. Wang, Y. Wang, C. Jiang, F. Gao, PLOS ONE 10 (2015) e0125701.

[48] L.Wang,Y.Han, S.Zhou,X.Guan,Biosensors and Bioelectronics 62(2014)158-162.

[49] Y. Long, C. Zhou, C. Wang, H. Cai, C. Yin, Q. Yang, C. James, D. Xiao, Scientific Reports 6 (2016) 23949-23958.

[50] J. Lee, M.-H. Hong, S. Han, J. Na, I. Kim, Y.-J. Kwon, Y.-b. Lim, H.-J. Choi, Nanoscale Research Letters 11 (2016) 341-347.

[51] B. Li, Z.l. Li, B. Situ, Z. Dai, Q. Liu, Q. Wang, D. Gu, L. Zheng, Biosensors andBioelectronics 52 (2014) 330-336.

[52] D. Zhang, Y. Peng, H. Qi, Q. Gao, C. Zhang, Biosensors and Bioelectronics 25 (2010) 1088-1094. 